\title{
Las zonas neurogénicas en el adulto y su relación con las enfermedades neuropsiquiátricas
}

\author{
Gerardo Ramírez-Rodríguez, ${ }^{1}$ María del Carmen Silva-Lucero, ${ }^{1,2}$ Laura Gómez-Virgilio, 1,2 \\ María del Ángel Ocaña-Fernández,' Leonardo Ortiz-López, ${ }^{1}$ Mario O. Torres-Pérez, ${ }^{1}$ \\ Marco Antonio Meraz-Ríos ${ }^{2}$
}

Artículo original

\section{SUMMARY}

Neuropsychiatric diseases (NPD) are characterized by changes in brain plasticity involving alterations in the morphology and functionality of neurons. However, affectations of the neuronal development (neurogenesis) in the adult brain are also shown.

The neurogenic process is widely regulated by different factors such as genes, microenvironment, hormones, neurotransmitters, environmental cues and, also, nutrition. Thus, alterations in these factors negatively impact the neuronal development.

Several studies performed in humans have revealed alterations of neurogenesis in NPD. However, most of the knowledge derives from studies done in animal models of NPD. The evidences from animal models are controversial, thus the use of human-induced pluripotent stem cells as a model of NPD has marked a way to study alterations in the neuronal development. Recently, the use of another cellular model for studying NPD has been proposed. Multipotent stem cells derived from olfactory epithelium (MOESCs) are a good candidate. However, evidences are scarce and deeper studies are necessary to know if there is or not a correlation of alterations in neuronal development in the OE with the changes observed in the brain; or if the MOESCs can mimic alterations shown in NPD that could let to get more knowledge about the factors promoting these diseases.

Thus, in this review we discuss basic information about adult neurogenesis under physiological and non-physiological conditions in the hippocampus, olfactory bulb and olfactory epithelium.

Key words: Neurogenesis, neuropsychiatric disorders, aging; stem cells.

\section{RESUMEN}

Las enfermedades neuropsiquiátricas (ENP) se caracterizan por cambios en la plasticidad cerebral que incluyen la pérdida neuronal en regiones específicas en el encéfalo, cambios en la transmisión sináptica originada por alteraciones en los contactos sinápticos y también por la expresión de genes. Además, otro proceso que forma parte de la plasticidad cerebral y que también se encuentra afectado en las ENP es la generación de nuevas neuronas (neurogénesis).

El proceso neurogénico en el adulto es regulado de manera fina por diversos factores como los aspectos genéticos, celulares, el microambiente, los elementos neuroquímicos, los ambientales y los nutricionales. Las alteraciones de estos factores impactan en el desarrollo y en la función de las nuevas neuronas.

Algunos estudios realizados en humanos han revelado las alteraciones en la neurogénesis en algunos ENP. Sin embargo los mayores avances logrados han utilizado modelos animales de ENP. En algunos casos estas evidencias son controvertidas y recientemente se han tratado de aclarar utilizando cultivos de células madre pluripotenciales-inducibles humanas como modelos de ENP. Otro modelo que se ha propuesto para estudiar las alteraciones en el desarrollo neuronal en las ENP son las células madre multipotenciales del epitelio olfatorio (CMPEO). Sin embargo las evidencias obtenidas con las CMPEO son escasas y resulta necesario demostrar si existe o no un correlato con las alteraciones que ocurren en el desarrollo neuronal a nivel central en las ENP, o bien si las CMPEO pueden mostrar las alteraciones observadas en las ENP que permitan obtener información acerca de los factores que promueven estas enfermedades.

Por lo tanto en esta revisión se incluyen aspectos básicos de la neurogénesis e información relevante de las alteraciones de este proceso en las tres regiones neurogénicas en el adulto: el hipocampo, el bulbo olfatorio y el epitelio olfatorio.

Palabras clave: Neurogénesis, enfermedades neuropsiquiátricas, envejecimiento, células madre.

Laboratorio de Neurogénesis, Subdirección de Investigaciones Clínicas, Instituto Nacional de Psiquiatría Ramón de la Fuente Muñiz.

2 Departamento de Biomedicina Molecular. Centro de Investigación de Estudios Avanzados (CINVESTAV). IPN.

Correspondencia: Dr. Gerardo Ramírez-Rodríguez. Laboratorio de Neurogénesis. Division de Investigaciones Clínicas, Instituto Nacional de Psiquiatría Ramón de la Fuente Muñiz. Calz. México-Xochimilco 101, San Lorenzo Huipulco, Tlalpan, 14370 México, DF. Tel. 52 (55) 4160 - 5053. E-mail: gbernabe@imp.edu.mx 


\section{INTRODUCCIÓN}

La formación de neuronas nuevas obedece a un mecanismo que se encuentra finamente modulado y que responde tanto a factores intrínsecos como extrínsecos. ${ }^{1,2}$

Contrariamente a lo que se había pensado acerca de la naturaleza estática del cerebro adulto, ahora se sabe que el encéfalo es capaz de generar neuronas nuevas que pueden integrarse en los circuitos neuronales existentes para desempeñar funciones especializadas como el aprendizaje y la memoria, o bien permitir la adaptación a circunstancias novedosas pero también complejas. ${ }^{3-6}$

Los primeros hallazgos que indicaron la formación de nuevas neuronas derivan de los estudios realizados por Joseph Altman en 1966, quien reportó la presencia de células con capacidad proliferativa en el cerebro adulto. ${ }^{7}$ Este trabajo sentó las bases para el estudio de la regeneración neuronal. ${ }^{7}$

Además del hipocampo, otra región del cerebro en donde se lleva a cabo la generación de neuronas de manera constitutiva es el bulbo olfatorio. ${ }^{8,9}$ Ambas regiones pueden estar afectadas en las ENP. ${ }^{10-14}$ De manera interesante algunas ENP también cursan con alteraciones de la regeneración neuronal que se lleva a cabo en el epitelio olfatorio (EO), región de la cavidad nasal en la que, al igual que el hipocampo y el bulbo olfatorio, el proceso neurogénico se presenta de manera constitutiva. ${ }^{15-21}$

La regeneración neuronal de las tres regiones se debe a la presencia de células multipotenciales que responden a diferentes estímulos para llevar a cabo la formación de nuevas neuronas. ${ }^{8,18,22-24}$

\section{NEUROGÉNESIS EN EL CEREBRO ADULTO: EL HIPOCAMPO}

El hipocampo es una estructura del sistema límbico que participa en el procesamiento de la memoria y es una de las tres regiones en donde se lleva a cabo la generación de neuronas de manera constitutiva. ${ }^{4,5,7}$ En esta estructura, específicamente en el giro dentado (GD), las neuronas nuevas derivan de las células madre que se localizan en la zona subgranular (ZSG)(figura 1). Una vez que las células madre se dividen dan lugar a las células que se amplifican rápidamente. Estas últimas van a migrar tangencialmente para empezar a diferenciarse en neuronas, las cuales van a sobrevivir al desarrollar dendritas que se proyectan hacia la capa molecular ${ }^{25}$ (figura 1).

Dado que el hipocampo es una estructura esencial para la formación de memoria de tipo espacial ${ }^{4,5} \mathrm{y}$ también para memorias relacionadas con las emociones, se ha considerado que la neurogénesis hipocámpica tiene un papel importante en la formación y regulación de conductas emotivas y de aprendizaje. ${ }^{4,5}$ En este sentido, y con base en estudios neuroanatómicos, computacionales, electrofisiológicos, conductuales e imagenológicos se ha sugerido un papel crucial para las nuevas neuronas del hipocampo en la formación de memorias episódicas. ${ }^{26}$

\section{NEUROGÉNESIS EN EL CEREBRO ADULTO: EL BULBO OLFATORIO}

El bulbo olfatorio mantiene una organización fundamental de cinco capas: la del nervio olfatorio, la glomerular, la plexiforme externa, la de células mitrales y la capa de célu-
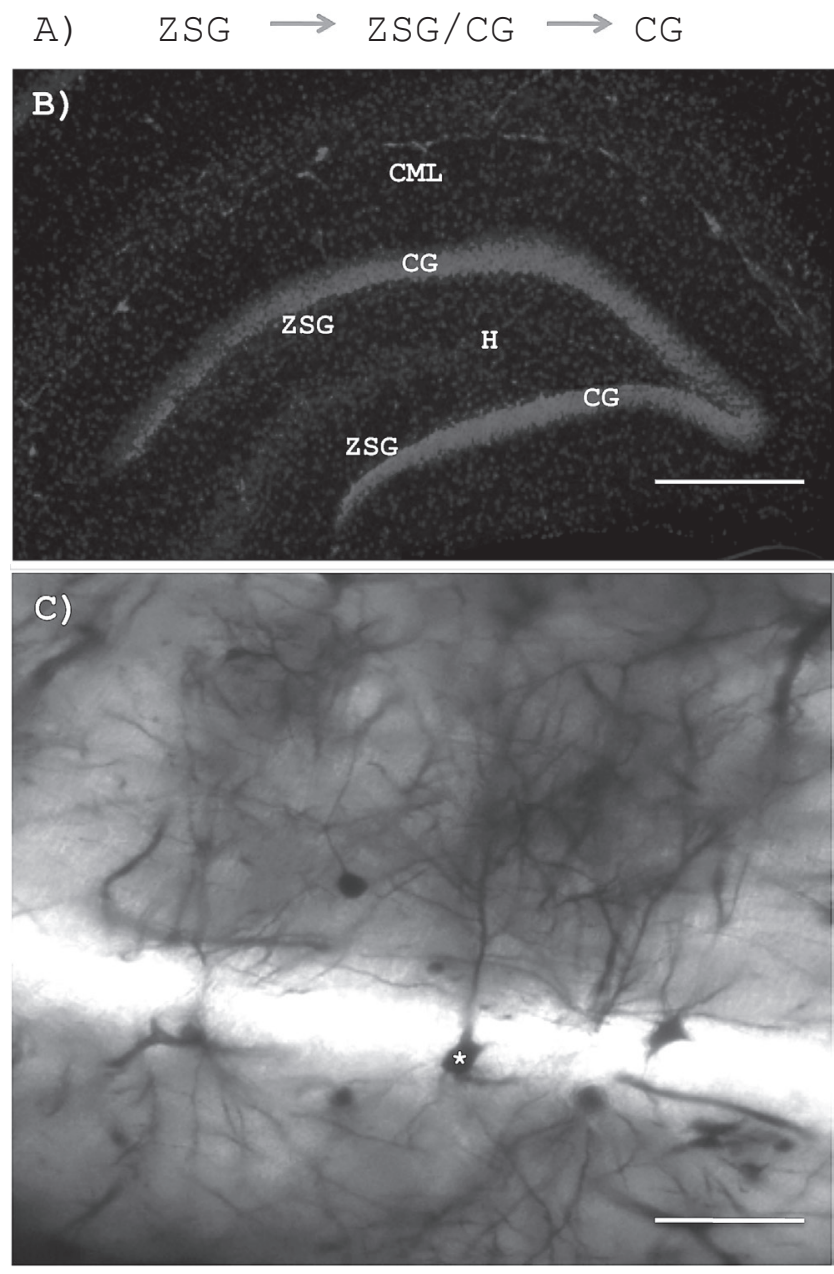

Figura 1. La zona neurogénica en el giro dentado del hipocampo. El panel A muestra una secuencia de las áreas a través de las cuales las células madre y los precursores neuronales migran hasta alcanzar la capa granular (CG), lugar donde se integran las nuevas neuronas en el hipocampo. Lo anterior se muestra claramente en el panel $B$, en una micrografía de un corte coronal teñido con un marcador nuclear. La micrografía muestra la zona subgranular (ZSG), la capa molecular (CML) y la zona hilar del giro dentado. La micrografía fue tomada con un microscopio de fluorescencia Nikon Eclipse Ti. El panel $C$, muestra neuronas de la CG del GD que fueron impregnadas con metales utilizando la técnica de Golgi-Cox. El asterisco indica una célula granular con proyecciones dendríticas hacia la CML. La micrografía fue tomada con un microscopio de campo claro de Leica DM500. La barra de calibración en B corresponde 300 micrómetros, y en $\mathrm{C}$ a 60 micrómetros. 
las granulares. Los axones no mielinizados de las neuronas sensoriales bipolares, localizadas en el epitelio olfatorio, se fasciculan y penetran al bulbo olfatorio para formar la capa del nervio olfatorio. Las terminales de estas fibras hacen sinápsis con las dendritas descendentes de las células mitrales en el glomérulo. Las células mitrales son las neuronas de proyección eferentes primarias del bulbo olfatorio y, junto con las células penacho, inervan el núcleo olfatorio anterior y se extienden hacia el tracto olfatorio directamente a las cortezas olfatorias primarias ${ }^{2}$ (figura 2).

Las nuevas neuronas del bulbo olfatorio derivan de los neuroblastos que provienen de las células madre que residen en la zona subventricular (ZSV), en los ventrículos laterales $^{8,27}$ (figura 2). El proceso neurogénico en el bulbo olfatorio se inicia con la división de las células madre para dar origen a los neuroblastos. Estos progenitores neuronales migrarán en grupos sobre la cadena migratoria rostral hasta alcanzar el bulbo olfatorio, lugar en donde se lleva a cabo su diferenciación terminal. Una vez en el bulbo olfatorio, las células de nueva generación formarán interneuronas granulares, pero también neuronas periglomerulares. ${ }^{2}$ De este modo las interneuronas generadas reemplazan a las células granulares viejas para mantener íntegra la circuitería del bulbo olfatorio. ${ }^{28}$ Esto es interesante, ya que indica que la neurogénesis es importante para el mantenimiento de la capacidad olfativa y también para la discriminación de olores. ${ }^{15}$ Considerando lo anterior, el bulbo olfatorio presenta un papel relevante al procesar la señal olfatoria con respecto a su contexto, señal que es transmitida hasta el bulbo olfatorio por las neuronas sensoriales localizadas en el epitelio olfatorio $^{19,20}$ (figura 2).

\section{NEUROGÉNESIS EN EL EPITELIO OLFATORIO}

El epitelio olfatorio es análogo al neuroepitelio del tubo neural, a partir del cual se desarrolla el cerebro en el embrión. ${ }^{29}$ Este epitelio se encuentra en la placa cribiforme, el septum nasal y en las porciones medias y superiores de los turbinados, en el adulto.

Histológicamente el epitelio olfatorio es un tejido heterogéneo compuesto de neuronas olfatorias bipolares y multiciliadas, células con microvellosidades, células basales identificadas como los progenitores de las neuronas sensoriales del epitelio olfatorio, y células sustentaculares. ${ }^{15,30}$ Las neuronas olfatorias presentan axones no mielinizados que forman haces, llamados fila olfatoria, para cruzar el foramen en la placa cribiforme y se proyectan hacia regiones glomerulares específicas en el bulbo olfatorio. ${ }^{15,17,30}$

El epitelio olfatorio es considerado como una tercera zona neurogénica, pero externa al cerebro, ya que presenta la generación constitutiva de neuronas sensoriales que se lleva a cabo por los progenitores neuronales residentes en el
A)

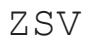
CMR
$\mathrm{BO}$ EO

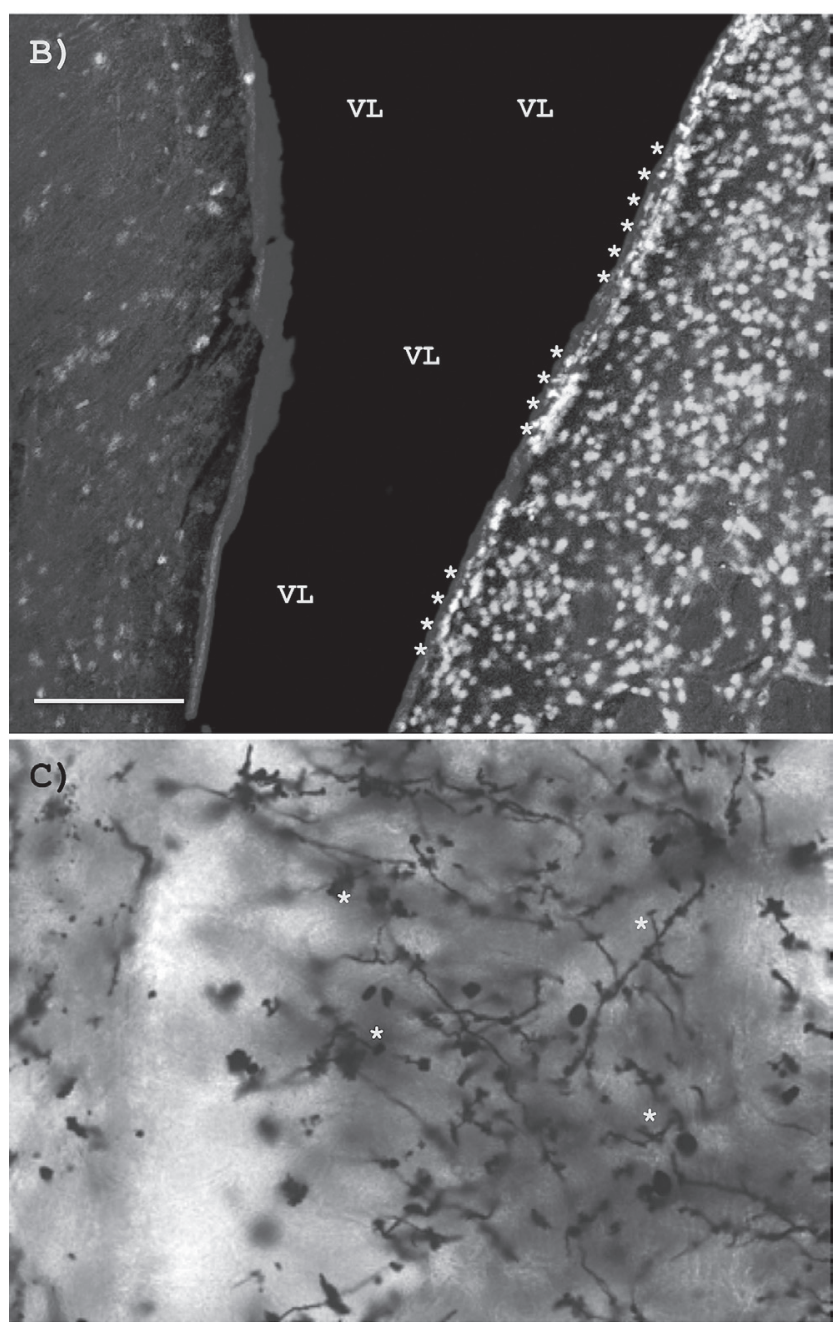

Figura 2. La zona neurogénica en la zona subventricular/bulbo olfatorio (ZSV/BO). El panel A muestra el proceso para la generación de nuevas neuronas en la ZSV/BO. Además, se incluye a la cadena migratoria rostral (CMR) que sirve de guía para que los neuroblastos migren hasta alcanzar el bulbo olfatorio (BO), lugar en donde las nuevas neuronas recibirán las proyecciones provenientes de las neuronas sensoriales del epitelio olfatorio (EO). El panel B muestra los ventrículos laterales (VL) y células en estado de proliferación (asteriscos). La micrografía fue adquirida con un microscopio de fluorescencia Nikon Eclipse Ti y la barra de calibración es $=200$ micrómetros. En el panel $C$, se muestran neuronas del bulbo olfatorio (asteriscos) que fueron impregnadas con metales utilizando la técnica de Golgi-Cox. Algunas células muestran en sus dendritas espinas dendríticas. La micrografía fue adquirida con un microscopio de campo claro de Leica DM500.

mismo epitelio. ${ }^{17,18}$ Diversos trabajos han mostrado la regulación molecular y el direccionamiento celular para llevar a cabo la neurogénesis en el epitelio olfatorio a lo largo de la vida. ${ }^{17,20,31}$ La regeneración continua producida por el reemplazo de las neuronas sensoriales es regulada por los mismos factores de crecimiento o por sustancias neuroquímicas que promueven el desarrollo del cerebro tanto en su etapa embrionaria como en su etapa adulta. ${ }^{17,20,31}$ 
Las células del epitelio olfatorio, tanto de roedores como de humanos, presentan progenitores neuronales que expresan la proteína nestina que pertenece a la familia de los filamentos intermedios, los cuales sirven de sostén para la estructura celular; y también presentan redes microtubulares formadas por la proteína específica de neuronas llamada tubulina tipo III. De tal modo que estas células son las responsables de la regeneración de la población neuronal sensorial del epitelio olfatorio a lo largo de la vida y cuya población es la encargada de transmitir la señal olfativa hasta el cerebro a través del bulbo olfatorio ${ }^{17,20,31}$ (figura 2).

\section{ALTERACIONES EN EL DESARROLLO NEURONAL EN EL ADULTO Y EN LAS ENFERMEDADES NEUROPSIQUIÁTRICAS}

Diversos estudios realizados en algunos ENP han mostrado que existe una correlación entre ellas y las alteraciones del desarrollo neuronal que se lleva a cabo en las zonas neurogénicas. ${ }^{10,11,13,31-35}$ En este sentido, a continuación revisaremos información relevante de las alteraciones del proceso neurogénico causadas por factores como el estrés y también sobre las alteraciones que están presentes en diversos padecimientos neuropsiquiátricos, tales como las enfermedades de Parkinson, de Huntington, de Alzheimer y en la esquizofrenia. Estos padecimientos neuropsiquiátricos cursan con alteraciones específicas del proceso neurogénico en el GD del hipocampo, en el bulbo olfatorio y alguno de ellos también las presentan en el epitelio olfatorio.

\section{El estrés}

El hipocampo es una estructura del sistema límbico que se encuentra alterada tanto en su estructura como en su función en pacientes con trastornos neuropsiquiátricos. ${ }^{6}$ La alteración en el hipocampo también se ha observado en estudios preclínicos, en los que se han utilizado modelos animales de enfermedades neuropsiquiátricas. ${ }^{6}$ Entre los procesos que son afectados se encuentra a la neurogénesis hipocámpica. ${ }^{6}$

En relación a lo anterior, el estrés es un factor importante para la presencia de la ansiedad y para el desarrollo de la depresión mayor. Los estudios preclínicos muestran que la exposición a estresores afecta al proceso neurogénico. De manera interesante los estresores agudos afectan principalmente la proliferación de las células progenitoras del GD del hipocampo, sin afectar la diferenciación y la sobrevivencia. En cambio, el estrés crónico afecta tanto la proliferación celular como la diferenciación y sobrevivencia celulares. Estudios en humanos han revelado también que los efectos del estrés sobre el proceso neurogénico son revertidos por los fármacos antidepresivos, siendo éste el primer reporte que indica que la disminución de la proliferación celular en el cerebro humano puede ser revertida. Lo anterior apoya la hipótesis de que en las alteraciones de la plasticidad cerebral presentes en la depresión y la ansiedad la neurogénesis hipocámpica es uno de los factores que pueden estar siendo afectados. ${ }^{32,36-42}$ Esta información ha sido ampliamente revisada recientemente. ${ }^{43}$

\section{La esquizofrenia}

Ésta es una enfermedad neuropsiquiátrica que presenta alteraciones en el desarrollo neuronal temprano. ${ }^{44-48}$ Se trata de una ENP multifactorial y se ha sostenido que entre los factores que convergen para que se desarrolle se encuentran los genes y el medio ambiente, así como diferencias neuroquímicas y en la estructura del cerebro como los cambios en la plasticidad neuronal. ${ }^{31,45,46,49,50}$

Los primeros síntomas como las alucinaciones y los delirios son evidentes durante la pubertad y la primera juventud (entre los 16 y los 30 años); sin embargo en algunos casos la enfermedad se presenta después de los 45 años. ${ }^{45} \mathrm{El}$ diagnóstico o reconocimiento de los cambios progresivos es de relevancia debido a que gracias a la intervención terapéutica, en teoría, se pueden aliviar muchos de los síntomas. ${ }^{45}$

De manera interesante, a nivel celular se ha demostrado que no hay cambios en la proporción de células; sin embargo éstas se encuentran empacadas más densamente debido a una distrofia relativa de su arborización dendrítica y a cambios en el neurópilo. ${ }^{47}$ Lo anterior sugiere que los cambios en la estructura cerebral participan en el desarrollo de la esquizofrenia. Considerando que en la plasticidad neuronal se incluye a la neurogénesis, entonces es viable pensar que en ésta la producción de novo de neuronas, oligodendrocitos y astrocitos es adecuada; en cambio la inserción de los nuevos elementos neuronales a las redes existentes puede estar alterada, en conjunto con la presencia de la poda sináptica, la mielinización y la anormal presencia de apoptosis. ${ }^{50}$

Entre los factores a nivel genético se ha planteado que la haplo-insuficiencia del gen que codifica para la proteína alterada en la esquizofrenia-1 (DISC1, por sus siglas en inglés) es uno de los factores de riesgo para la esquizofrenia que mejor se han establecido. ${ }^{51}$ La represión de la señalización de DISC1 conduce a un desarrollo dendrítico acelerado en células neuronales de nueva generación, así como un incremento en la migración, lo cual origina una integración inadecuada de estas nuevas células a la red neuronal. ${ }^{52}$

Las alteraciones en la neuroplasticidad presentes en la esquizofrenia también pueden ser debidas a las cascadas de señalización de algunos factores de crecimiento. Las evidencias clínicas indican que la vía del factor de crecimiento transformante (TGF- $\beta$, por sus siglas en inglés) se encuentra hiperactivada, mientras que la vía de Wnt se encuentra hipoactiva. Los resultados obtenidos con el análisis de estas vías de señalización celular muestran una migración y una diferenciación aceleradas, lo cual afecta la inserción correcta de las nuevas neuronas. ${ }^{50}$ Otra vía de señalización implica- 
da en la patofisiología de la esquizofrenia es la retinoide, ${ }^{53,54}$ que juega un papel central en los procesos de neurodesarrollo temprano como la neurogénesis. ${ }^{54,55}$ Recientemente se describió un aumento en la expresión de un receptor retinoide en las células granulares del hipocampo.$^{55}$ Dicho receptor funciona como un interruptor para controlar la transición de los eventos de proliferación a diferenciación de las células madre durante el desarrollo. La expresión aumentada de este receptor en las células granulares del giro dentado durante la esquizofrenia puede ser consecuencia de un desarrollo temprano defectuoso o de procesos neurogénicos alterados en el cerebro adulto. ${ }^{56}$

Los pacientes con esquizofrenia también presentan un aumento en la cavidad de los ventrículos laterales, lugar en donde se encuentran las células madre que formarán nuevas neuronas en el bulbo olfatorio ${ }^{56,57}$ (figura 2). Esto podría estar afectando la formación de neuronas en el bulbo olfatorio y, de algún modo, también podría estar alterada la neurogénesis en el epitelio olfatorio. ${ }^{56}$

En relación a lo anterior, en explantes del epitelio olfatorio de pacientes con esquizofrenia, se ha encontrado un aumento en la tasa de proliferación y en la de muerte celular. De tal modo que la proliferación celular alterada en el epitelio olfatorio concuerda con los cambios tanto en el número de precursores neuronales como en el de neuronas olfatorias de individuos con esquizofrenia. Estos resultados sugieren una disregulación en la neurogénesis olfatoria. ${ }^{58}$

Además de DISC-1, también la expresión de los transcritos de RAD51L1, NCK2 y VIPR1 se incrementa en la esquizofrenia..$^{29}$ Los niveles de expresión de las proteínas codificadas por los genes NCK2 y VIPR1 se relacionan directamente con la proliferación celular alterada en las células del epitelio olfatorio; mientras que RAD51L1 fosforila a la ciclina E, cdk2 y p53 y de este modo afecta la sincronización de la fase G1 del ciclo celular. De manera inversa, se ha visto que la expresión de dos genes involucrados en la neurogénesis (PTN, NTF5) y otro gen involucrado en la diferenciación neuronal (NPDC1) se encuentra disminuida en las células del epitelio olfatorio. La expresión reducida de genes concernidos en la diferenciación neuronal también es consistente con la observación de un aumento en la proliferación celular debido a que, por ejemplo, NDPC1 se expresa solamente en células neurales cuando detienen su división y comienzan a diferenciarse. ${ }^{29}$ En conjunto, en la esquizofrenia se ha mostrado que existen alteraciones en el proceso neurogénico tanto del hipocampo como del bulbo y del epitelio olfatorio.

\section{Enfermedad de Parkinson}

Se trata de un trastorno del sistema motor común entre las personas de edad avanzada, caracterizado por temblores, rigidez, entre otros síntomas que van a interferir con las actividades diarias de los pacientes. En la EP las neuronas dopaminérgicas de la sustancia nigra pars compacta, que es un ganglio basal del mesencéfalo, se degeneran selectivamente. Estas neuronas se proyectan hacia las neuronas GABAérgicas en el estriado y participan en el movimiento muscular coordinado. Adicionalmente, las células colinérgicas en el núcleo basal, el sistema serotonérgico del núcleo del raphé, en la amígdala, el hipocampo, en el bulbo olfatorio, en la corteza temporal y en la del cíngulo presentan degeneración celular. ${ }^{13,59} \mathrm{La}$ EP parece no estar asociada con alguna mutación genética hereditaria, sin embargo diferentes genes han sido propuestos como factores para su desarrollo. Tampoco ha sido aclarado el mecanismo que lleva a la muerte neuronal. ${ }^{13,59-61}$

En la etiología de la EP se ha propuesto la participación de factores ambientales como el estilo de vida, las toxinas y también el envejecimiento, así como ciertos factores genéticos. Estudios realizados en modelos animales basados en la inducción de daño con neurotoxinas han mostrado alteraciones en el proceso neurogénico, específicamente en los eventos de proliferación y en la supervivencia celular. ${ }^{59,62}$

De manera interesante se ha visto una disminución significativa en la proliferación celular en la ZSV de pacientes con EP y también en modelos animales de la misma enfermedad ${ }^{59}$ En modelos de EP con neurotoxinas se observa un aumento en el número de interneuronas dopaminérgicas en la capa glomerular del bulbo olfatorio. Del mismo modo, estudios en pacientes con la EP muestran un aumento en neuronas dopaminérgicas. ${ }^{13,62}$

Considerando lo anterior es necesario realizar estudios preclínicos que indiquen el grado de afectación no sólo en la sustancia nigra, sino también los posibles cambios en regeneración neuronal presentes en el epitelio olfatorio, ya que también se han descrito alteraciones en la capacidad olfativa de los pacientes con EP. ${ }^{63}$ En este sentido, recientemente se han aislado células precursoras de pacientes con EP, modelo con el cuál se pretende abordar las alteraciones presentes en la neurogénesis en el epitelio olfatorio y también para obtener información acerca de la complejidad genética y de las interacciones ambientales que contribuyen al desarrollo de algunas enfermedades neuropsiquiátricas. ${ }^{64} \mathrm{~A}$ pesar de que se han propuesto éste y otros modelos celulares (figura 3), es necesario establecer si las células aisladas tienen la capacidad de mostrar los cambios que se encuentran a nivel central en todas las enfermedades neuropsiquiátricas, o bien sólo en aquellas que cursan con alteraciones o pérdida de la capacidad olfativa.

\section{Enfermedad de Huntington}

Se trata de una enfermedad de herencia autosómica dominante con síntomas progresivos que incluyen movimientos involuntarios, déficits cognitivos y alteraciones psiquiátricas. La patofisiología más notable de la enfermedad es la degeneración progresiva de las proyecciones de las neuronas y una gliosis aumentada, llevando a una atrofia marcada del estriado, el cual es adyacente a la zona subventricular. 

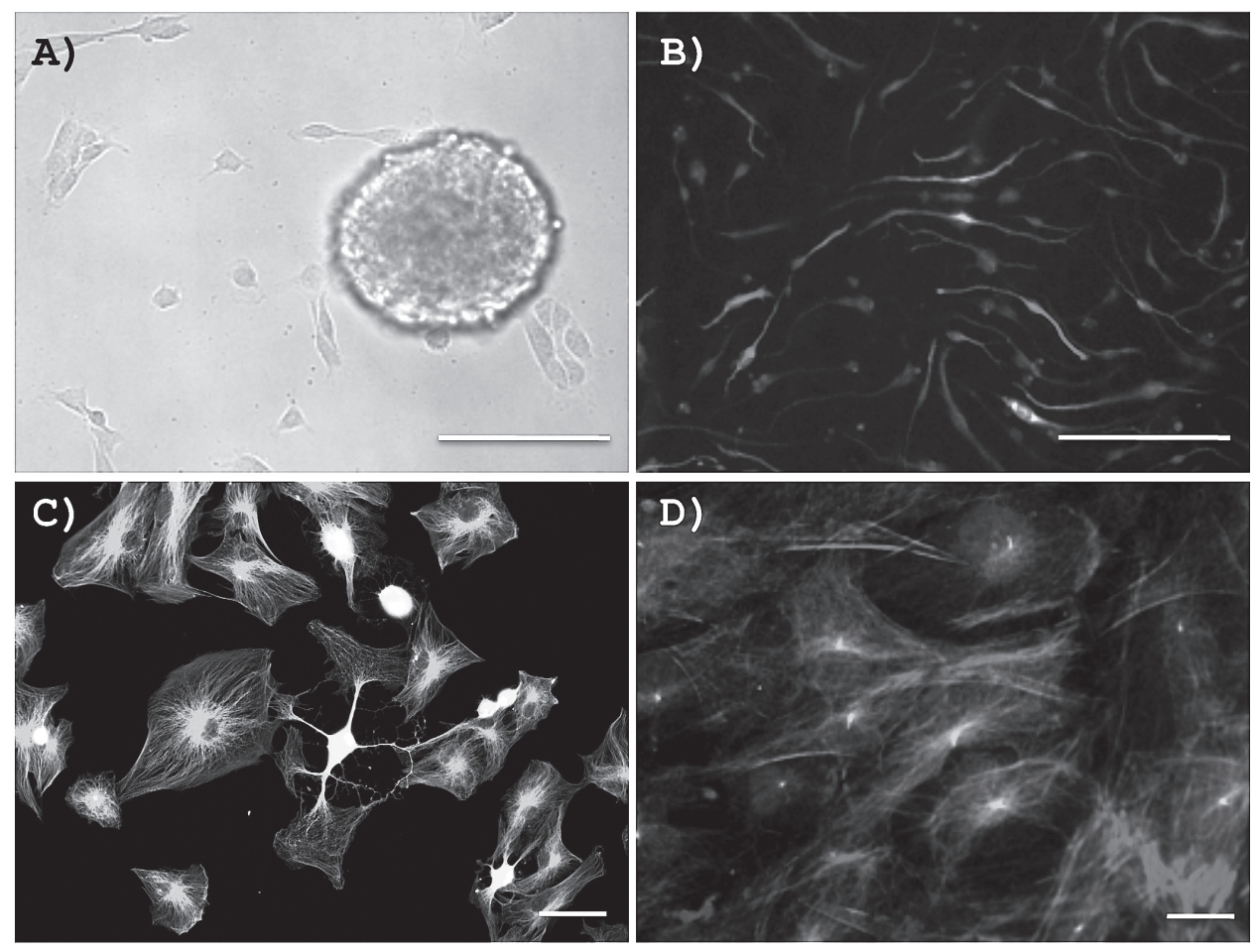

Figura 3. Las células madre y los precursores de las tres zonas neurogénicas. En el planel A se muestra un agregado de células formando una neuroesfera debido a la capacidad proliferativa. El planel B muestra células diferenciadas a partir de las células madre localizadas en la zona subgranular del giro dentado del hipocampo. El panel C, muestra células aisladas de los ventrículos laterales y que dan origen a las neuronas del bulbo olfatorio. El panel $D$, muestra células madre aisladas del epitelio olfatorio. La barra de calibración en los páneles $A$ y $B=80$ micrómetros; mientras que en $C=30$ micrómetros y en $D=15$ micrómetros. Todas las imágenes fueron adquiridas con un microscopio de fluorescencia Nikon Eclipse Ti.

La EH es causada por una expansión de repetidos CAG en el gen que codifica para la proteína hungtintina. ${ }^{25}$ Esta proteína es citoplasmática y se asocia con los microtúbulos y vesículas. La proteína participa en el tráfico de organelos. ${ }^{65,66}$ Recientemente, se ha reportado que la hungtintina- 1 es la responsable de la generación de gradientes de factores de crecimiento, nutrientes y neurotransmisores, promoviendo la homeostasis en el líquido cefalorraquídeo. ${ }^{65,66}$

El análisis post-mortem de cerebros de pacientes con la EH ha mostrado que la zona subventricular se vuelve más gruesa, con un aumento en la proliferación celular, mientras que el número de células de amplificación transitoria y neuroblastos aumenta moderadamente. ${ }^{67}$ Esta alteración en la neurogénesis ha sido descrita en el modelo de ratón transgénico de la EH, los ratones R6/2, los cuales portan los genes humanos de la $\mathrm{EH}$ con los repetidos alargados $\mathrm{CAG} .{ }^{68}$

En los roedores R6/ 2 se ha visto un aumento en la capacidad de autorrenovación de la población de células madre de la ZSV, lo cual ocurre en paralelo con el progreso de la enfermedad. Además, los neuroblastos y las neuronas de nueva generación migran hacia el estriado de estos ratones. Aunado a lo anterior, la migración de los neuroblastos hacia el bulbo olfatorio se inhibe de manera significativa. Esto es interesante ya que impacta en la generación de nuevas neuronas que se deben localizar en el bulbo olfatorio, de tal modo que la discriminación de olores por parte de los pacientes con EH y también en el modelo animal (roedores R6/1) se encuentra perturbada, quizás debido a la disminución en el reemplazo de neuronas en esta estructura. ${ }^{69}$ Lo anterior también puede ser afectado por un ensamblaje erróneo del cilio, ya que se ha demostrado que la deleción del gen que codifica para la huntingtina en las células ependimales de la ZSV altera la formación del cilio primario, por lo que afecta negativamente la migración de los neuroblastos. ${ }^{65,66}$

En el mismo sentido, y considerando las alteraciones en la discriminación de olores y la disminución en la generación de nuevas neuronas en el bulbo olfatorio, se puede sugerir que la formación de neuronas sensoriales en el epitelio olfatorio también está alterada; de tal modo que todo el sistema que participa en la olfación (epitelio olfatorio-bulbo olfatorio) quedaría lesionado en la $\mathrm{EH}$.

\section{Enfermedad de Alzheimer}

Esta enfermedad neuropsiquiátrica presenta degeneración neuronal progresiva caracterizada por una disminución 
gradual en la memoria y en la ejecución de funciones corticales superiores. Es la forma más común de demencia y representa aproximadamente del 60 al 70\% de todos los casos. Las características neuropatológicas de la enfermedad incluyen atrofia, pérdida neuronal, formación de marañas neurofibrilares y placas seniles. ${ }^{13,33,35,62,70}$

En 1907, Aloïs Alzheimer describió el caso de una paciente de 51 años de edad que presentaba un cuadro de demencia con una grave desorientación y alucinaciones. El estudio microscópico del cerebro de esta mujer permitió descubrir en el interior de las células la existencia de lesiones en forma de agregados insolubles, a los cuales este autor denominó degeneración neurofibrilar, la que coexistía con placas neuríticas. El descubrimiento de la historia clínica de esta paciente ha permitido el desarrollo y progreso del estudio de la enfermedad. ${ }^{13,31,33,62}$

Estudios realizados en humanos que presentaban la EA mostraron un aumento en la neurogénesis. ${ }^{70}$ En este caso se midió la expresión de proteínas marcadoras de neuronas inmaduras que sugieren la formación de nuevas neuronas en el hipocampo de pacientes con EA. En relación a los controles, los cerebros con enfermedad de Alzheimer mostraron un incremento en la expresión de DCX y TUC-4 en la ZSG del GD del hipocampo. Otro estudio demostró que en pacientes con la variedad presenil hay una mayor proliferación en las capas CA1-3, posiblemente reflejando los cambios gliales y vasculares asociados, pero no neurogénesis. ${ }^{32}$ En contraste con los resultados realizados en tejido humano post-mortem, la neurogénesis adulta se encuentra disminuida en el modelo de ratón transgénico de la enfermedad de Alzheimer (ratones TgCRND8), los cuales sobreexpresan la proteína precursora amiloide humana (APP). ${ }^{71}$

La acumulación extracelular del péptido $\beta$-amiloide es un evento importante en la patogénesis de EA. La mayoría de los estudios se han enfocado en los mecanismos en donde el péptido induce la degeneración de neuronas adultas de tal modo que la infusión intracerebroventricular del péptido causa un daño en la neurogénesis en la ZSV del ratón adulto. Además, se ha demostrado que la presencia de las formas oligoméricas del péptido $A \beta$, ya sea $A \beta_{1-40}$ y/o $A \beta_{1-42}$ las cuales se producen como consecuencia del procesamiento amiloidogénico de la proteína precursora amiloide (APP), tiene un efecto en la neurogénesis de las dos regiones del cerebro, la ZSG del GD y en la ZSV de las paredes del tercer ventrículo. Lo anterior ocurre mucho antes de la formación, tanto de las placas amiloides como de las marañas neurofibrilares y de la pérdida neuronal, todas ellas características de la enfermedad.

En relación a la disfunción olfatoria, las anormalidades en la olfacción en la EA incluyen una disminución en el umbral de detección del olor, déficits marcados en la habilidad para identificar olores y daños en la memoria de reconocimiento de los mismos. En un meta-análisis de estudios de umbral de detección de olor, identificación olfatoria y me- moria de reconocimiento, se encontraron déficit en los tres dominios en la EA. Esto puede indicar una participación tanto de las estructuras olfatorias "periféricas" (por ejemplo, el neuroepitelio olfatorio) responsable de la detección del olor como de las regiones cerebrales olfatorias "centrales" (por ejemplo, el bulbo olfatorio, el núcleo olfatorio anterior, la corteza prepiriforme, la amígdala, la corteza entorrinal, el prosencéfalo basal) responsables de la identificación y la memoria olfatorias. ${ }^{31,33,35}$

Diversos estudios han examinado la pérdida celular y su degeneración, las marañas neurofibrilares y las placas seniles en el bulbo olfatorio de pacientes con la EA. El núcleo olfatorio anterior es el que presenta las marañas neurofibrilares y sólo en algunos casos las células mitrales, las de penacho y las granulares externas las presentan también. Asímismo se ha reportado la degeneración y la pérdida de células mitrales en el núcleo olfatorio anterior. Además de esto, también se han observado placas seniles en este núcleo olfatorio. ${ }^{31}$ De tal manera que las cantidades de neuronas en el núcleo olfatorio anterior estuvieron reducidas sustancialmente en los pacientes con la EA, en comparación con los controles. ${ }^{33}$

En relación con la zona neurogénica localizada en el epitelio olfatorio, en 1989 se describió la presencia de una nueva forma de patología neurítica en los pacientes con EA, así como cantidades disminuidas de neuronas olfatorias sensoriales. Se realizaron estudios subsecuentes para determinar la presencia de las características neuropatológicas de la EA como las marañas neurofibrilares y las placas seniles. Se intentó además descubrir si la expresión de epítopes particulares de la proteína tau permiten distinguir las diversas etapas de la enfermedad. Mientras que las marañas neurofibrilares no se observaron en el EO de algunos de los casos estudiados, la inmunorreactividad de tau fue evidente en las neuritas distróficas. ${ }^{31,33}$

En contraste con los estudios anteriores, al obtenerse por biopsia mucosa nasal de pacientes con EA se describió inmunorreactividad a la proteína tau en las dendritas y en el soma de las neuronas olfatorias, de tal modo que se observaron depósitos de placas extracelulares inmunorreactivas a la tau e inmunorreactividad a la ubiquitina dendrítica. Los hallazgos anormales fueron más comunes en los casos de EA pero también estuvieron presentes en los casos control. La disfunción olfatoria observada en pacientes con Alzheimer se encuentra directamente relacionada con la presencia de agregados amorfos, intracelulares y vesiculares del péptido $\mathrm{A} \beta$ así como con la expresión de una tau hiperfosforilada con una apariencia típica de la enfermedad como las marañas neurofibrilares, ambas en el epitelio olfatorio. Por otro lado, las neuronas olfatorias de pacientes con Alzheimer desarrollan, de igual manera, patología asociada a la enfermedad incluyendo pérdida neuronal, gran número de marañas neurofibrilares y placas amiloides. Los cultivos de dichas neuronas han mostrado elementos de la patofisiolo- 
gía cerebral de la EA como el procesamiento anormal de la proteína precursora amiloide. ${ }^{13,31,33}$

Debido a que en las neuronas provenientes del epitelio olfatorio se presenta un procesamiento anormal de la proteína amiloide, de manera análoga a lo que ocurre en el cerebro y que igualmente desarrolla la patología relacionada con la enfermedad, es factible asumir que la presencia de las formas oligoméricas de $\mathrm{A} \beta$ en las células neuronales del epitelio olfatorio, las cuales tienen una capacidad de regeneración, tengan un efecto sobre su potencial neurogénico y que en cierta medida influyan de manera directa en la disfunción olfatoria observada en los pacientes con EA.

\section{Epilepsia}

Además de los trastornos neuropsiquiátricos antes mencionados, también la epilepsia, un trastorno neurológico, tiene fuertes vínculos con la estructura y la función del hipocampo, en particular con su neurogénesis. ${ }^{72}$ Por ejemplo, la intensa actividad convulsiva aumenta la proliferación de las células en la ZSG, lo que provoca la formación de un mayor número de neuronas. ${ }^{62}$

Estos resultados son particularmente interesantes porque las convulsiones neonatales parecen estar asociados con defectos a largo plazo sobre la sensibilidad a las convulsiones, la cognición y el volumen del hipocampo. ${ }^{73}$

Además de lo anterior, también se ha reportado que tanto la actividad convulsiva breve como la prolongada reducen el número de células granulares de nueva generación cuando se analiza el proceso al día postnatal 17 y después de una semana de que ocurrió la convulsión. Estos resultados ayudaron a comprender cómo es que las convulsiones neonatales afectan el desarrollo neuronal y la formación de circuitos neuronales. ${ }^{74}$ Este trabajo se suma a la creciente apreciación de que la respuesta neurogénica a la actividad convulsiva es dependiente de la edad, hecho que es relevante para entender la mayor incidencia de aparición de convulsiones ya sea en la niñez o en la edad adulta tardía. Por ejemplo, mientras que la actividad inducida experimentalmente en la edad adulta aumenta la proliferación en la ZSG, la actividad convulsiva en la vejez no se asocia con un aumento de ésta. ${ }^{75}$ Las convulsiones también pueden producir la migración y la morfología aberrante de las neuronas nuevas lo que se acompaña de alteraciones en la función sináptica, ${ }^{76}$ por lo que la neurogénesis aberrante es probablemente una de las muchas anormalidades del hipocampo que contribuyen a la epilepsia y/o a la disfunción cognitiva.

\section{CONCLUSIONES}

En este trabajo revisamos información relevante que apoya la presencia de la generación de neuronas de manera constitutiva en tres zonas neurogénicas durante la etapa adulta: el hipocampo, el bulbo olfatorio y el epitelio olfatorio.
En conjunto, todas las evidencias aquí mencionadas nos muestran que las alteraciones en el proceso neurogénico son relevantes, aunque no de manera exclusiva, para causar el desarrollo de las enfermedades neuropsiquiátricas y neurológicas. Interesantemente, en algunas de estas enfermedades como las enfermedades de Hungtington y de Alzheimer y en la esquizofrenia los cambios en el proceso neurogénico se presentan en las tres zonas, lo cual nos habla de alteraciones que ocurren en los sistemas involucrados en la memoria y la percepción de olores, evento en el que también participa la formación de memoria. Esto puede explicar los déficits y las alteraciones en ambas funciones en algunas enfermedades neuropsiquiátricas.

Es importante destacar que los cambios en el desarrollo neuronal presentes en las enfermedades neuropsiquíatricas han sido evidenciados en modelos animales y, algunos, en tejido humano post-mortem. Sin embargo, a la fecha no se tiene un panorama completo debido a la naturaleza multifactorial de estos padecimientos.

En un intento por generar mayor información acerca de las alteraciones en el desarrollo neuronal, presentes en estas enfermedades neuropsiquiátricas, se han utilizado modelos celulares derivados de pacientes con alguna enfermedad específica. Estos modelos reflejan cambios de la región neurogénica a partir de la cual se aíslan las células, sin mostrar, hasta la fecha, los cambios que ocurren en otras regiones, y sólo han indicado una correlación con la presencia de alguna enfermedad neuropsiquiátrica. Lo anterior es entendible, sobre todo porque cada región neurogénica desarrolla neuronas especializadas para una función específica.

Finalmente, consideramos que el estudio del proceso neurogénico es relevante ya que es uno de los factores que están alterados en las enfermedades neuropsiquiátricas, y debido a la función especializada de cada tipo neuronal formado en las zonas neurogénicas en el adulto se puede obtener información acerca de los cambios en el desarrollo neuronal en cada zona neurogénica y su posible relación con alguna enfermedad. Algunos estudios que desarrolla nuestro grupo están encaminados a conocer y entender la biología celular de las células madre de origen neuronal presentes en la etapa adulta, para una futura aplicación (figura 3). Pretendemos entender el impacto de las alteraciones en el proceso neurogénico para el desarrollo de algunas enfermedades neuropsiquiátricas y/o el beneficio de la estimulación de la neurogénesis para retrasar los efectos del envejecimiento sobre la plasticidad neuronal.

\section{AGRADECIMIENTOS}

Este trabajo fue apoyado parcialmente por el INPRFM y por el CONACYT (101316 y 119182). MCSL y LGV son apoyadas por el CONACYT a través del Programa de Posgrado del Departamento de Biomedicina Molecular del CINVESTAV. MAOF y MTP son apoyados por el CONACYT (101316) y por la SSA-PROBEI, respectivamente. 


\section{REFERENCIAS}

1. Kempermann G, Jessberger S, Steiner B et al. Milestones of neuronal development in the adult hippocampus. Trends Neurosci 2004;27:447-452.

2. Lledo PM, Alonso M, Grubb MS. Adult neurogenesis and functional plasticity in neuronal circuits. Nat Rev Neurosci 2006;7:179-193.

3. Kempermann G, Wiskott L, Gage FH. Functional significance of adult neurogenesis. Curr Opin Neurobiol 2004;14:186-191.

4. Aimone JB, Deng W, Gage FH. Adult neurogenesis: integrating theories and separating functions. Trends Cogn Sci 2010;14:325-337.

5. Deng W, Aimone JB, Gage FH. New neurons and new memories: how does adult hippocampal neurogenesis affect learning and memory? Nat Rev Neurosci 2010;11:339-350.

6. Kempermann G, Krebs J, Fabel K. The contribution of failing adult hippocampal neurogenesis to psychiatric disorders. Curr Opin Psychiatry 2008;21:290-295.

7. Altman J, Das GD. Autoradiographic and histological studies of postnatal neurogenesis. I. A longitudinal investigation of the kinetics, migration and transformation of cells incorporating tritiated thymidine in neonate rats, with special reference to postnatal neurogenesis in some brain regions. J Comp Neurol 1966;126:337-389.

8. Alvarez-Buylla A, Garcia-Verdugo JM. Neurogenesis in adult subventricular zone. J Neurosci 2002;22:629-634.

9. van den Berge SA, Middeldorp J, Zhang CE et al. Longterm quiescent cells in the aged human subventricular neurogenic system specifically express GFAP-delta. Aging Cell 2010;9:313-326.

10. Curtis MA, Faull RL, Eriksson PS. The effect of neurodegenerative diseases on the subventricular zone. Nat Rev Neurosci 2007;8:712-723.

11. Curtis MA, Low VF, Faull RL. Neurogenesis and progenitor cells in the adult human brain: a comparison between hippocampal and subventricular progenitor proliferation. Dev Neurobiol 2012;72:990-1005.

12. May VE, Nuber S, Marxreiter F et al: Impaired olfactory bulb neurogenesis depends on the presence of human wild-type alpha-synuclein. Neuroscience 2012;222:343-355.

13. Winner B, Kohl Z, Gage FH. Neurodegenerative disease and adult neurogenesis. Eur J Neurosci 2011;33:1139-1151.

14. Winner B, Regensburger M, Schreglmann S et al. Role of alpha-synuclein in adult neurogenesis and neuronal maturation in the dentate gyrus. J Neurosci 2012;32:16906-16916.

15. Enwere E, Shingo T, Gregg $C$ et al. Aging results in reduced epidermal growth factor receptor signaling, diminished olfactory neurogenesis, and deficits in fine olfactory discrimination. J Neurosci 2004;24:8354-8365.

16. Aimone JB, Deng W, Gage FH. Resolving new memories: a critical look at the dentate gyrus, adult neurogenesis, and pattern separation. Neuron 2011;70:589-596.

17. Feron F, Bianco J, Ferguson I et al. Neurotrophin expression in the adult olfactory epithelium. Brain Res 2008;1196:13-21.

18. Girard SD, Deveze A, Nivet E et al: Isolating nasal olfactory stem cells from rodents or humans. J Vis Exp 2011;54:e2762; doi:10,3791/2762.

19. MacDonald KP, Murrell WG, Bartlett PF et al. FGF2 promotes neuronal differentiation in explant cultures of adult and embryonic mouse olfactory epithelium. J Neurosci Res 1996;44:27-39.

20. Mackay-Sima A, Chuahb MI. Neurotrophic factors in the primary olfactory pathway. Prog Neurobiol 2000;62:527-559.

21. Manceur AP, Tseng M, Holowacz T et al: Inhibition of glycogen synthase kinase- 3 enhances the differentiation and reduces the proliferation of adult human olfactory epithelium neural precursors. Exp Cell Res 2011;317:2086-2098.

22. Babu H, Cheung G, Kettenmann $H$ et al: Enriched monolayer precursor cell cultures from micro-dissected adult mouse dentate gyrus yield functional granule cell-like neurons. PLoS One 2007;2:e388.

23. Gage FH, Kempermann G, Palmer TD et al: Multipotent progenitor cells in the adult dentate gyrus. J Neurobiol 1998;36:249-266.

24. Palmer TD, Willhoite AR, Gage FH. Vascular niche for adult hippocampal neurogenesis. J Comp Neurol 2000;425:479-494.
25. Snell RG, MacMillan JC, Cheadle JP et al. Relationship between trinucleotide repeat expansion and phenotypic variation in Huntington's disease. Nat Genet 1993;4:393-397.

26. Sahay A, Scobie KN, Hill AS et al. Increasing adult hippocampal neurogenesis is sufficient to improve pattern separation. Nature 2011;472:466-470.

27. Lim DA, Alvarez-Buylla A. Interaction between astrocytes and adult subventricular zone precursors stimulates neurogenesis. Proc Natl Acad Sci U S A 1999;96:7526-7531.

28. Ninkovic J, Pinto L, Petricca $S$ et al. The transcription factor Pax6 regulates survival of dopaminergic olfactory bulb neurons via crystallin alphaA. Neuron 2010;68:682-694.

29. McCurdy RD, Feron F, Perry $C$ et al. Cell cycle alterations in biopsied olfactory neuroepithelium in schizophrenia and bipolar I disorder using cell culture and gene expression analyses. Schizophr Res 2006;82:163-173.

30. Mackay-Sim A. Concise review: patient-derived olfactory stem cells: new models for brain diseases. Stem Cells 2012;30:2361-2365.

31. Arnold SE, Smutzer GS, Trojanowski JQ et al. Cellular and molecular neuropathology of the olfactory epithelium and central olfactory pathways in Alzheimer's disease and schizophrenia. Ann N Y Acad Sci 1998;855:762-775.

32. Boekhoorn K, Joels M, Lucassen PJ. Increased proliferation reflects glial and vascular-associated changes, but not neurogenesis in the presenile Alzheimer hippocampus. Neurobiol Dis 2006;24:1-14.

33. Esiri MM, Wilcock GK. The olfactory bulbs in Alzheimer's disease. J Neurol Neurosurg Psychiatry 1984;47:56-60.

34. Mirochnic S, Wolf S, Staufenbiel M et al. Age effects on the regulation of adult hippocampal neurogenesis by physical activity and environmental enrichment in the APP23 mouse model of Alzheimer disease. Hippocampus 2009;19:1008-1018.

35. Sohrabi HR, Bates KA, Weinborn MG et al: Olfactory discrimination predicts cognitive decline among community-dwelling older adults. Transl Psychiatry 2012;2:e118.

36. Caspi A, Sugden K, Moffitt TE et al. Influence of life stress on depression: moderation by a polymorphism in the 5-HTT gene. Science 2003;301:386-389.

37. Coe CL, Kramer M, Czeh B et al. Prenatal stress diminishes neurogenesis in the dentate gyrus of juvenile rhesus monkeys. Biol Psychiatry 2003;54:1025-1034.

38. Gould E, Tanapat P. Stress and hippocampal neurogenesis. Biol Psychiatry 1999;46:1472-1479.

39. Mirescu C, Gould E. Stress and adult neurogenesis. Hippocampus 2006;16:233-238.

40. Mirescu C, Peters JD, Gould E. Early life experience alters response of adult neurogenesis to stress. Nat Neurosci 2004;7:841-846.

41. Perera TD, Coplan JD, Lisanby SH et al. Antidepressant-induced neurogenesis in the hippocampus of adult nonhuman primates. J Neurosci 2007;27:4894-4901.

42. Pittenger C, Duman RS. Stress, depression, and neuroplasticity: a convergence of mechanisms. Neuropsychopharmacology 2008;33:88-109.

43. Ramírez-Rodríguez G, Laguna-Chimal J, Ortiz-López L et al. Los fármacos antidepresivos como reguladores de la neurogénesis hipocámpica de roedores y humanos adultos. Salud Mental 2011;34:497-506.

44. Goldberg TE, Weinberger DR, Berman KF et al. Further evidence for dementia of the prefrontal type in schizophrenia? A controlled study of teaching the Wisconsin Card Sorting Test. Arch Gen Psychiatry 1987;44:1008-1014.

45. McGrath JJ, Feron FP, Burne TH et al: The neurodevelopmental hypothesis of schizophrenia: a review of recent developments. Ann Med 2003;35:86-93.

46. Raz S, Raz N, Weinberger DR et al. Morphological brain abnormalities in schizophrenia determined by computed tomography: a problem of measurement? Psychiatry Res 1987;22:91-98.

47. Selemon LD, Goldman-Rakic PS. The reduced neuropil hypothesis: a 
circuit based model of schizophrenia. Biol Psychiatry 1999;45:17-25.

48. Weinberger DR. Implications of normal brain development for the pathogenesis of schizophrenia. Arch Gen Psychiatry 1987;44:660-669.

49. Berman KF, Weinberger DR, Shelton RC et al. A relationship between anatomical and physiological brain pathology in schizophrenia: lateral cerebral ventricular size predicts cortical blood flow. Am J Psychiatry 1987;144:1277-1282.

50. Kalkman HO. Altered growth factor signaling pathways as the basis of aberrant stem cell maturation in schizophrenia. Pharmacol Ther 2009;121:115-122.

51. Porteous DJ, Thomson P, Brandon NJ et al: The genetics and biology of DISC1--an emerging role in psychosis and cognition. Biol Psychiatry 2006;60:123-131.

52. Duan X, Chang JH, Ge S et al. Disrupted-In-Schizophrenia 1 regulates integration of newly generated neurons in the adult brain. Cell 2007;130:1146-1158.

53. Goodman AB. Three independent lines of evidence suggest retinoids as causal to schizophrenia. Proc Natl Acad Sci U S A 1998;95:7240-7244.

54. Maden M. Retinoid signalling in the development of the central nervous system. Nat Rev Neurosci 2002;3:843-853.

55. Rioux L, Arnold SE. The expression of retinoic acid receptor alpha is increased in the granule cells of the dentate gyrus in schizophrenia. Psychiatry Res 2005;133:13-21.

56. Toro CT, Deakin JF. Adult neurogenesis and schizophrenia: a window on abnormal early brain development? Schizophr Res 2007;90:1-14.

57. Austin CP, Ky B, Ma L et al: Expression of Disrupted-In-Schizophrenia1, a schizophrenia-associated gene, is prominent in the mouse hippocampus throughout brain development. Neuroscience 2004;124:3-10.

58. Feron F, Perry C, Hirning MH et al. Altered adhesion, proliferation and death in neural cultures from adults with schizophrenia. Schizophr Res 1999;40:211-218.

59. Hoglinger GU, Rizk P, Muriel MP et al. Dopamine depletion impairs precursor cell proliferation in Parkinson disease. Nat Neurosci 2004;7:726-735.

60. Borta A, Hoglinger GU. Dopamine and adult neurogenesis. J Neurochem 2007;100:587-595.

61. Winner B, Geyer M, Couillard-Despres S et al. Striatal deafferentation increases dopaminergic neurogenesis in the adult olfactory bulb. Exp Neurol 2006;197:113-121.
62. Steiner B, Wolf S, Kempermann G. Adult neurogenesis and neurodegenerative disease. Regen Med 2006;1:15-28.

63. Kaneko N, Sawamoto K. Adult neurogenesis and its alteration under pathological conditions. Neurosci Res 2009;63:155-164.

64. Murrell W, Wetzig A, Donnellan M et al. Olfactory mucosa is a potential source for autologous stem cell therapy for Parkinson's disease. Stem Cells 2008;26:2183-2192.

65. Keryer G, Pineda JR, Liot G et al. Ciliogenesis is regulated by a huntingtin-HAP1-PCM1 pathway and is altered in Huntington disease. J Clin Invest 2011;121:4372-4382.

66. Liu JP, Zeitlin SO. The long and the short of aberrant ciliogenesis in Huntington disease. J Clin Invest 2011;121:4237-4241.

67. Curtis MA, Penney EB, Pearson AG et al. Increased cell proliferation and neurogenesis in the adult human Huntington's disease brain. Proc Natl Acad Sci U S A 2003;100:9023-9027.

68. Batista CM, Kippin TE, Willaime-Morawek $S$ et al. A progressive and cell non-autonomous increase in striatal neural stem cells in the Huntington's disease R6/2 mouse. J Neurosci 2006;26:10452-10460.

69. Lazic SE, Grote H, Armstrong RJ et al. Decreased hippocampal cell proliferation in R6/1 Huntington's mice. Neuroreport 2004;15:811-813.

70. Jin K, Peel AL, Mao XO et al. Increased hippocampal neurogenesis in Alzheimer's disease. Proc Natl Acad Sci U S A 2004;101:343-347.

71. Herring A, Ambree $\mathrm{O}$, Tomm $\mathrm{M}$ et al. Environmental enrichment enhances cellular plasticity in transgenic mice with Alzheimer-like pathology. Exp Neurol 2009;216:184-192.

72. Parent JM. Adult neurogenesis in the intact and epileptic dentate gyrus. Prog Brain Res 2007;163:529-540.

73. Holmes GL, Gairsa JL, Chevassus-Au-Louis N et al. Consequences of neonatal seizures in the rat: morphological and behavioral effects. Ann Neurol 1998;44:845-857.

74. Porter BE. Neurogenesis and epilepsy in the developing brain. Epilepsia 2008;49(Supl 5):50-54.

75. Rao MS, Hattiangady B, Shetty AK. Status epilepticus during old age is not associated with enhanced hippocampal neurogenesis. Hippocampus 2008;18:931-944.

76. Jakubs K, Nanobashvili A, Bonde $S$ et al. Environment matters: synaptic properties of neurons born in the epileptic adult brain develop to reduce excitability. Neuron 2006;52:1047-1059.

Artículo sin conflicto de intereses 